\title{
Mesoporous drug carrier systems for enhanced delivery rate of poorly water-soluble drug: nimodipine
}

\author{
Anna Kiwilsza $^{1,2}$ - Bartłomiej Milanowski ${ }^{3}$ Kacper Drużbicki ${ }^{1,4}$ - L. Emerson Coy $^{2}$ • \\ Mikołaj Grzeszkowiak $^{1,2} \cdot$ Marcin Jarek $^{2} \cdot$ Jadwiga Mielcarek $^{5} \cdot$ Janina Lulek $^{3}$ • \\ Aleksandra Pajzderska ${ }^{1} \cdot$ Jan Wąsicki ${ }^{1,2}$
}

Published online: 29 March 2015

(c) The Author(s) 2015. This article is published with open access at Springerlink.com

\begin{abstract}
Two mesoporous silica materials: MCM-41 and SBA-15 were applied as potential nanocarriers for poorly soluble drug - nimodipine. Drug incorporation was performed using modified adsorption from the solution method and loaded samples before and after washing procedure were studied. The physical properties were verified by: differential scanning calorimetry, X-ray powder diffraction, electron microscopies (SEM/TEM) and Fourier-transform infrared spectroscopy (FT-IR). FT-IR results for bulk nimodipine were interpreted on the basis of first principles calculations (DFT). As a result of encapsulation process, in both matrices nimodipine appeared simultaneously in two forms: crystalline and amorphous, but the first one turned out to be easily removable during washing procedure. The in vitro dissolution and release
\end{abstract}

Electronic supplementary material The online version of this article (doi:10.1007/s10934-015-9955-3) contains supplementary material, which is available to authorized users.

Jan Wąsicki

jwasicki@amu.edu.pl

1 Faculty of Physics, Adam Mickiewicz University, Umultowska 85, 61-614 Poznan, Poland

2 NanoBioMedical Center, Adam Mickiewicz University, Umultowska 85, 61-614 Poznan, Poland

3 Department of Pharmaceutical Technology, Poznan University of Medical Sciences, Grunwaldzka 6, 60-780 Poznan, Poland

4 Frank Laboratory of Neutron Physics, Joint Institute for Nuclear Research, 141980 Dubna, Russia

5 Department of Inorganic and Analytical Chemistry, Poznan University of Medical Sciences, Grunwaldzka 6, 60-780 Poznan, Poland tests were performed with ultra pure water under supersaturating conditions. The release rate of the amorphous nimodipine from mesoporous silica materials was at least 70 times higher than dissolution rate of bulk drug, thus revealed a potential usefulness of such carrier in future pharmaceutical applications in terms of delivery of poorly soluble drugs.

Keywords Nimodipine - Mesoporous silica - MCM-41 . SBA-15 - Dissolution $\cdot$ Release rate

\section{Introduction}

Nimodipine (NM) is a 1,4-dihydropyridine derivative, classified as a second generation calcium channel antagonist [1]. As it is highly lipophilic and able to permeate through the blood-brain barrier, it is often used for the treatment of blood circulatory disorders in the brain. When administered orally, NM is quickly absorbed from the gastrointestinal tract but-due to the poor solubility and first-pass effect in the liver-its bioavailability is very low [2]. Moreover, NM can occur in two polymorphic forms, labelled as I and II, differing i.a. in colour, melting point and water solubility [3]. As it has been shown, under ambient conditions these two forms can coexist for a long time, but in the presence of traces of solvents, form I (metastable) undergoes a transformation to form II (stable). Although form I has more attractive pharmacological properties (i.e. its solubility in water is twice higher), it has not been used separately in clinical practice. According to Riekes et al. [4]., it was found that most of the commercially available products contain metastable form I of NM in greatest proportion, but still at least $9 \%$ of stable form II is present in them. 
According to the biopharmaceutics classification system (BCS) it belongs to group II [5], and its bioavailability after oral administration is just $15.9 \%$ on average [6]. Thus, conventional therapy with NM in the form of tablets or capsules requires administration of frequently taken much higher doses [7]. For these reasons, the use of a carrier that would improve the solubility of NM in body fluids and enhance drug delivery, seems much promising. In the recent decades, nanospheres $[8-12,15,16,18]$ or microspheres $[11,13,14,17]$ were widely used as drug carriers. A few reports have been published on the use of liquid and solid microemulsions [19, 20], solid dispersions [21-23] or drug-loaded type systems based on cyclodextrins [24-26] and lipid [27-29] or phospholipid micelles [30, 31]. Nevertheless, all these systems have organic character, so are potentially sensitive to external factors such as temperature or $\mathrm{pH}$, and can drastically change their properties under the effect of these factors [32]. In order to eliminate the presence of additional substances in the system, some attempts have been made to improve the solubility of NM by reduction of its particle size. The nanocrystals of NM formed under high pressure and stabilised by lyophilisation have revealed much less beneficial release profiles than the crystalline form of NM, both in water and in Fasted State Simulated Intestinal Fluid (FaSSIF) [33].

In view of the above, much promising seems to be the use of inorganic materials for production of thermally and $\mathrm{pH}$ stable system permitting improved delivery of NM. As follows from hitherto studies, one of the most often used pharmaceutical carriers is porous silica [34]. Although not much attention has been paid to the systems with NM, some attempts at using the hexagonally ordered mesoporous material SBA-15 [35] and macroporous silica spheres PMS [36] confirmed that these materials have beneficial effect on the release rate of NM.

The aim of our study was to compare the structural properties and release profiles of NM incorporated into two mesoporous silica materials MCM-41 and SBA-15.

\section{Experimental procedure}

\subsection{Materials}

Nimodipine (NM) and MCM-15 were purchased at Sigma Aldrich (USA) and studied without further purification. SBA-15 was synthesised by the method proposed by Zhao et al. [37, 38]. Sorption properties of the mesoporous materials were evaluated by low-temperature nitrogen sorption-Supporting materials, Chapter 1.

\subsection{Nimodipine encapsulation}

The NM encapsulation into a mesoporous carrier MCM-41 or SBA-15 was performed by a method according-Supporting materials, Chapter 2.

Encapsulated materials were studied before (1) and after washing with dichloromethane (2) and were labelled respectively: NM-M-1; MN-M-2 (for NM and MCM-41) and NM-S-1; MN-S-2 (for NM and SBA-15).

\subsection{Physical mixtures preparation}

The two samples NM with MCM-41 and SBA-15 were labelled as NM-M and NM-S, respectively-Supporting materials, Chapter 3.

\subsection{Methods}

The physical properties of nimodipine-loaded samples were verified by differential scanning calorimetry (DSC), $\mathrm{X}$-ray powder diffraction (XRPD), electron microscopies (SEM/TEM) and Fourier-transform infrared spectroscopy (FT-IR). In particular, FT-IR results for bulk nimodipine were interpreted on the basis of first principles calculations (DFT). The dissolution and release processes was investigated using on-line closed loop semi-automated flowthrough cell dissolution system (SOTAX AG, Switzerland) using method described in the Ph. Eur. 8.0 [39]. Supporting materials, Chapter 4.

\section{Results and discussion}

\subsection{Thermal analysis: DSC}

The DSC method was applied to check the effect of encapsulation on thermal properties of NM and the silica matrix. The thermograms of NM, physical mixtures NM-M and NM-S as well as unwashed samples NM-M-1 and NM$\mathrm{S}-1$ showed endothermic peak with a maximum at $128{ }^{\circ} \mathrm{C}$. It was assigned to the melting of NM form I [3].

However, in the DSC thermograms of the washed samples (NM-M-2/NM-S-2), the peak assigned to NM melting was not observed. This result implies that NM left inside the pores after washing does not have a crystalline form. On the other hand, the peak in the DSC thermograms of the unwashed samples (NM-M-1/NM-S-1) should be interpreted as corresponding to NM localised on the external surface of the mesopores.

To sum up, the DSC analysis proved that in the unwashed samples (NM-M-1/NM-S-1) kind of a dual system 
is realised in which: part of NM-localised on the external surface of mesopores-is crystalline and easily washed away, while the other part of NM-localised inside the pores-does not show crystalline features. On the contrary, the washed samples (NM-M-2/NM-S-2) are devoid of the easily removable crystalline NM.

\subsection{Thermal analysis: TGA}

Quantitative analysis of NM incorporated into the mesoporous silica matrices MCM-41 and SBA-15 was performed on the basis of TGA results, presented in Fig. 1.

TGA curve recorded for NM (in the range from room temperature to $550{ }^{\circ} \mathrm{C}$ ), above $220{ }^{\circ} \mathrm{C}$, revealed one sharp decrease corresponding to a significant mass loss, interpreted as a result of the process of decomposition.

For MCM-41 the TGA curve revealed two decreases. The first one is observed from room temperature to about $100{ }^{\circ} \mathrm{C}$, corresponding to the mass loss of $1.6 \%$, and assigned to dehydration that is the release of water adsorbed on the surface. The second decrease takes place from about 100 to $1000{ }^{\circ} \mathrm{C}$ and corresponds to $2.2 \%$ mass loss. It is assigned to gradual condensation of the surface silanol groups, described as the process of dehydroxylation [40]. For SBA-15 the analogous mass losses related to dehydration and dehydroxylation were 4.9 and $3.1 \%$, respectively. As follows from these data, SBA-15 had 3 times greater water content and 1.5 times greater $\mathrm{OH}$ group content per area unit than MCM-41.

For all samples with incorporated NM (NM-M-1/NMM-2 and NM-S-1/NM-S-2) the thermograms below $130{ }^{\circ} \mathrm{C}$ showed a decrease caused by dehydration, corresponding to about $0.5 \%$ mass loss. This value was much smaller than those observed for the silicas MCM-41 and SBA-15. Moreover, TGA analysis permitted evaluation of $\mathrm{NM}$ loading efficiency for encapsulation process into the silica

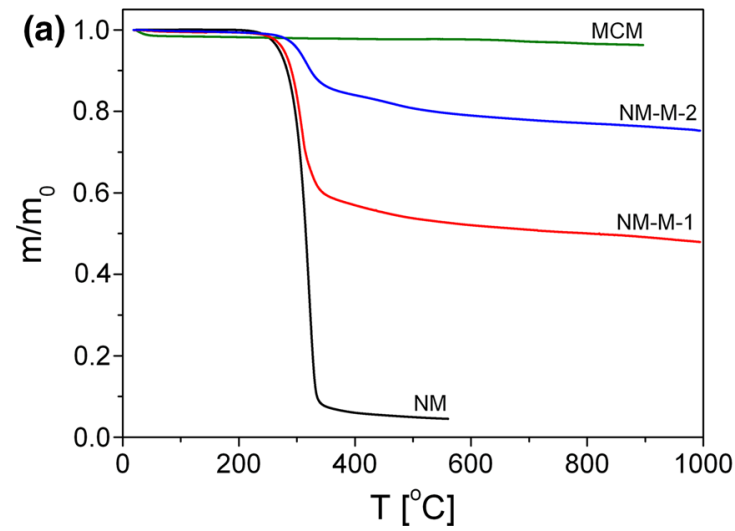

Fig. 1 TGA curves for: a nimodipine (NM), mesoporous silica (MCM) and two samples with incorporated NM: the unwashed one (NM-M-1) and washed one (NM-M-2); b nimodipine (NM), matrices. For the samples supported on MCM-41 it was $51.6 \%$ (NM-M-1) and $24.3 \%$ (NM-M-2), while for the samples supported on SBA-15: $50.5 \%$ (NM-S-1) and $17.9 \%$ (NM-S-2). So, the amount of NM incorporated in SBA-15 was smaller than that in MCM-41.

In general, assuming that in both silica matrices $\mathrm{NM}$ molecules (of the size smaller than $9 \AA$, Figure $1 \mathrm{~S}$-Supporting materials) are not sterically hindered during incorporation, and taking into account the mean pore size (Table 1S-Supporting materials), it can be estimated that the fulfillment of pores would imply formation of two layers of NM in MCM-41 and three layers of NM in SBA15 . In our case, assuming that in the washed samples (NMM-2/NM-S-2) drug is localised only inside the pores, the pore filling by NM molecules in the silica matrices was estimated to be $29 \%$ for MCM-41 and $20 \%$ for SBA- 15 . Considering these values in relation to the pore geometry and the size of the NM molecule, it can be concluded that the amount of incorporated NM was insufficient to make a single monolayer. For both matrices the mean coverage of the surface of silica pores was close to $60 \%$ and reached a slightly greater value for SBA-15.

\subsection{Powder X-ray diffraction: SAXS}

The effect of NM encapsulation on the structure of silica matrices was checked by the method of small angle X-ray scattering (SAXS). The spectra recorded for the silica matrices and samples with encapsulated NM are shown in Fig. 2.

The SAXS diffractograms of all samples contain three peaks characteristic for hexagonal arrangement of mesopores (space group p6 $\mathrm{mm}$ ), corresponding to the planes (100), (110) and (200) [37, 38]. In the diffractogram of MCM-41, the peaks are localised at higher angles than in that of SBA-15, which implies smaller lattice constant in

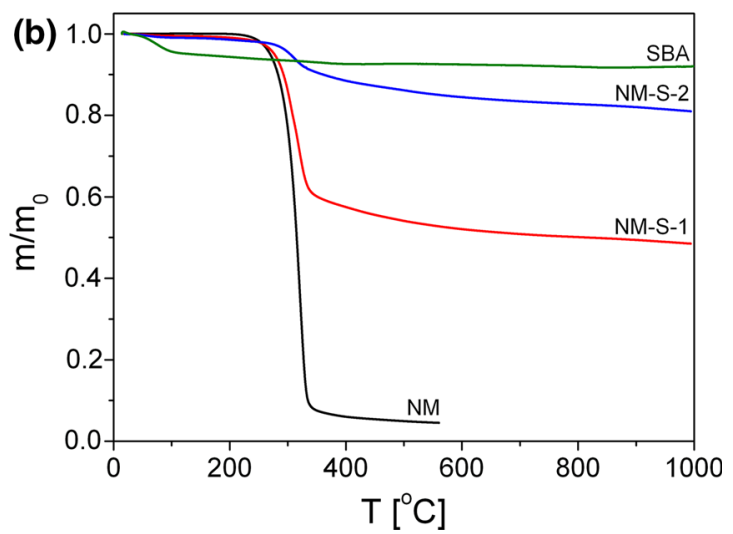

mesoporous silica (SBA) and two samples with incorporated NM: the unwashed one (NM-S-1) and washed one (NM-S-2) 
Fig. 2 SAXS diffractograms: a mesoporous silica (MCM) and samples with encapsulated NM: unwashed one (NM-M-1) and washed one (NM-M-2),

b mesoporous silica (SBA) and samples with encapsulated NM: unwashed one (NM-S-1) and washed one (NM-S-2)
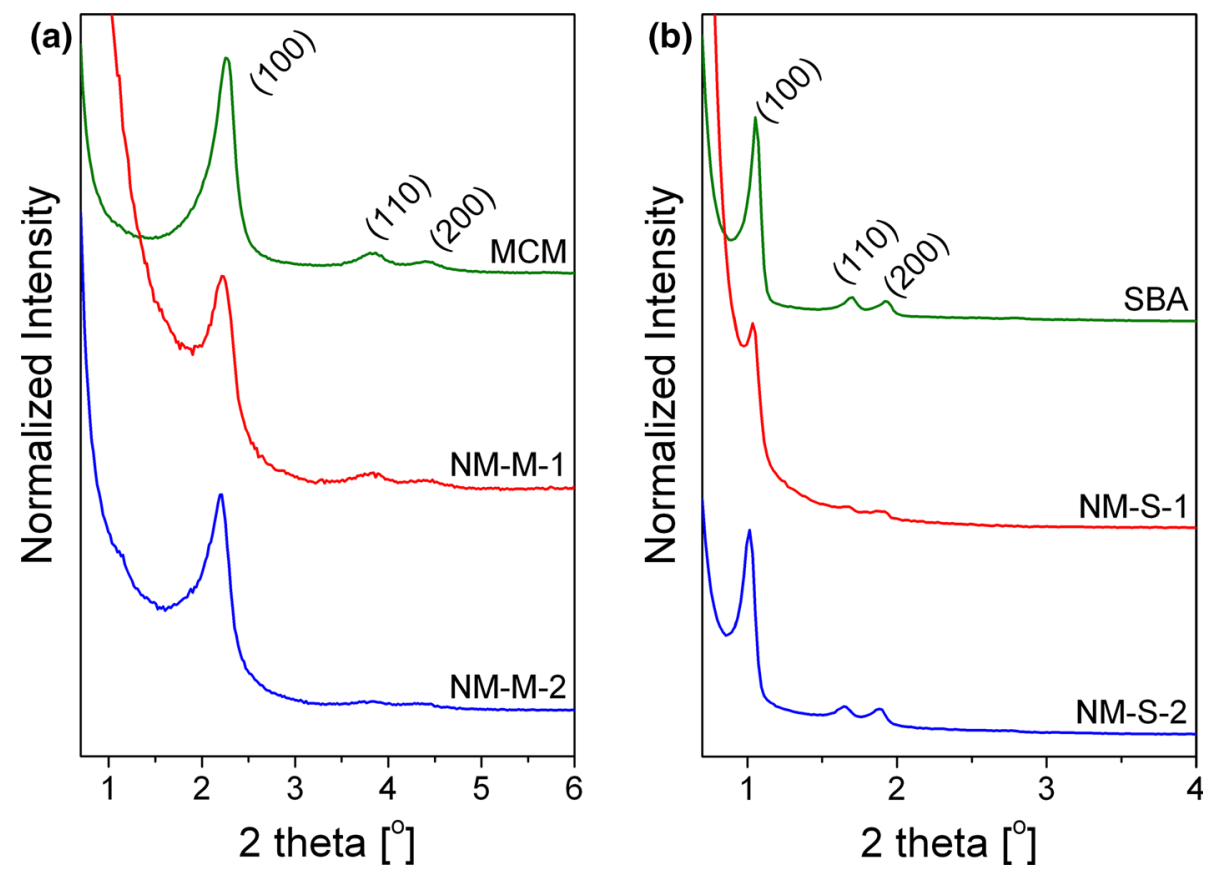

the former. In the diffractograms of the samples after NM encapsulation (NM-M-1/NM-M-2 and NM-S-1/NM-S-2) no changes in the shape or in position of the peaks were noted with respect to those in the diffractograms of silica matrices before encapsulation (MCM and SBA).

The interplanar distances $d(100)$, the lattice constants $a$ (calculated each time on the basis of the position of the most intense peak, that corresponds to the (100) plane) and the mean thicknesses of the pore wall $t$ (calculated taking into account the mean pore size, see Table 1S), are given in Table 2S-Supporting materials (Table 1).

As follows from Table 2, the agreement between the parameters characterising the porous structure before and after NM incorporation and washing, confirms that NM encapsulation has no effect on the structure of the silica matrix. In particular, it does not change the silica lattice parameters and does not degrade the hexagonal pore arrangement.

\subsection{Powder X-ray diffraction: WAXS}

All samples were also subjected to wide angle X-ray scattering study, the results of which are presented in Fig. 3.

According to literature, NM at room temperature can occur in two polymorphic forms [3]. The metastable form I is a racemic mixture, crystallizing in the monoclinic $\mathrm{P} 2{ }_{1} / \mathrm{c}$ space group, with the unit-cell built by four molecules. The form II is a conglomerate, which is also built by four nimodipine units, packed into the orthorhombic $\mathrm{P} 2{ }_{1} 2_{1} 2_{1}$ unit cell [41]. In both polymorphic forms, the nimodipine units are $\mathrm{N}-\mathrm{H} \cdots \mathrm{O}$ hydrogen-bonded, as the alkoxy groups in the flexible chains attract the protons from the dihydropyridine moieties. This weak hydrogen-bonding is characteristic for both polymorphs, while the subtle differences are mainly due to the van der Walls forces.

The WAXS diffractogram of NM (Fig. 3) shows peaks assigned to the monoclinic crystalline form I of NM, that is the metastable one [42]. The diffractograms of MCM-41 and SBA-15 silicas show no reflections assignable to crystalline ordering, so confirm the amorphous character of silica that builds the walls of both matrices, although they show a single broad peak with a maximum at $22^{\circ}$ in 2 theta scale [43]. The WAXS diffractograms of physical mixtures of NM and silicas (NM-M/NM-S) and those of unwashed samples with encapsulated NM (NM-M-1/NM-S-1) are superpositions of diffractograms of crystalline NM and the matrix. The diffractograms recorded for the washed samples with encapsulated NM (NM-M-2/NM-S-2) are devoid of the reflections characteristic of crystalline structure. Analysis of WAXS results indicates that, as a result of the process of encapsulation, NM is partly adsorbed on the silica surface in the crystalline form and this fraction of $\mathrm{NM}$ is removed on washing. Thus, the WAXS results confirm those obtained by DSC method (cf. Thermal analysis-DSC).

\subsection{Infrared spectroscopy studies}

\subsubsection{Vibrational analysis of nimodipine}

Although the title system has been studied extensively over the years, there is no report on vibrational analysis of NM 


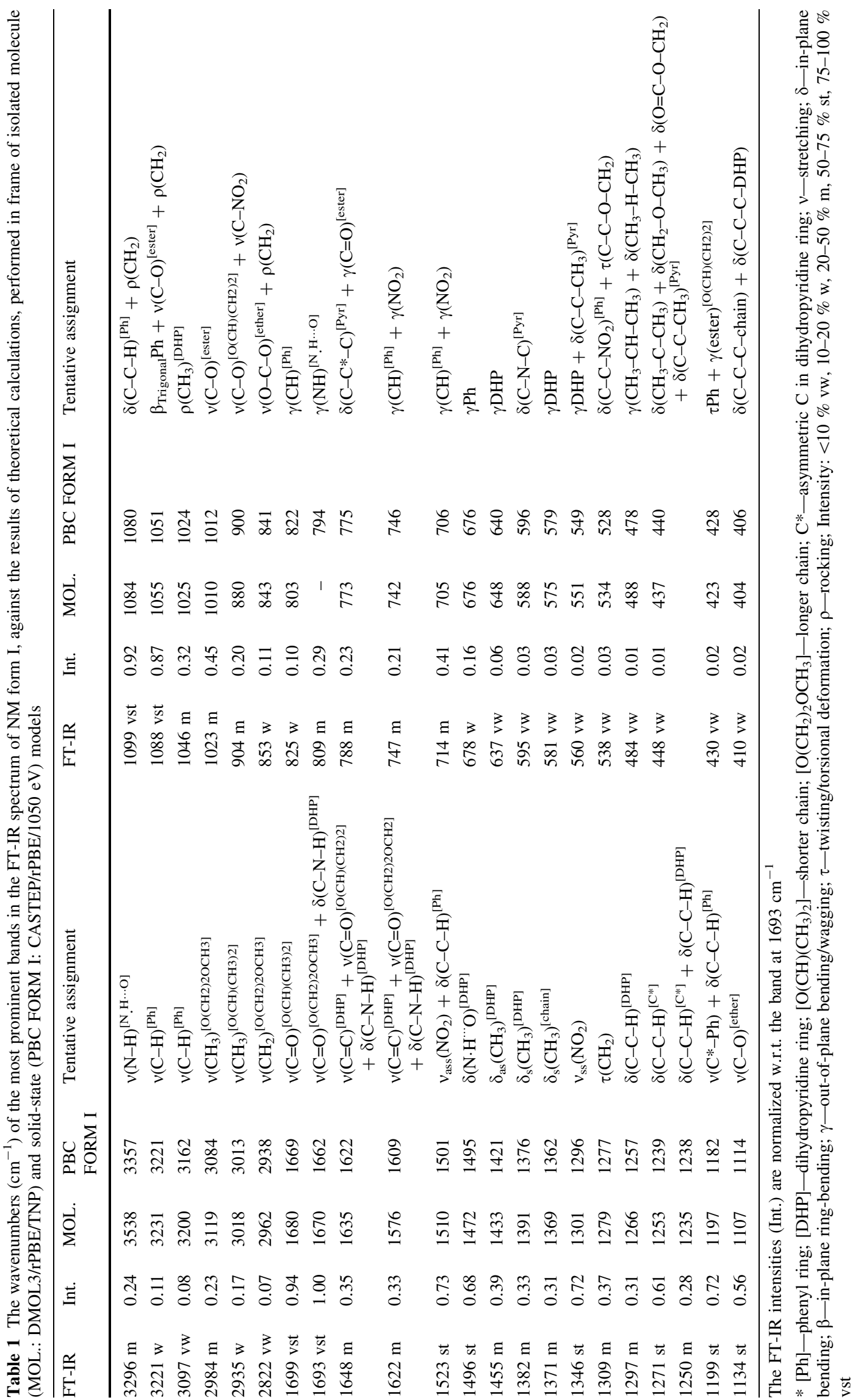


Table 2 Similarity factors $\left(\mathrm{f}_{2}\right)$ for NM dissolution and release profiles

\begin{tabular}{lccccccc}
\hline Tested powders $(n=3)$ & \multicolumn{7}{l}{ Similarity factor $\mathrm{f}_{2}$ value (profiles are similar when $\left.\mathrm{f}_{2}>50\right)$} \\
\cline { 2 - 8 } & $\mathrm{NM}$ & NM-M & NM-S & NM-M-1 & NM-S-1 & NM-M-2 & NM-S-2 \\
\hline NM & - & 69.28 & 50.87 & 48.80 & 47.84 & 5.03 & 6.19 \\
NM-M & 69.28 & - & 58.74 & 55.73 & 55.95 & 6.03 & 7.19 \\
NM-S & 50.87 & 58.74 & - & 75.50 & 75.26 & 7.62 & 8.92 \\
NM-M-1 & 48.80 & 55.73 & 75.50 & - & 80.16 & 8.04 & 9.43 \\
NM-S-1 & 47.84 & 55.95 & 75.26 & 80.16 & - & 8.23 & 9.54 \\
NM-M-2 & 5.03 & 6.03 & 8.92 & 8.04 & 8.23 & - & 51.30 \\
NM-S-2 & 6.19 & 7.19 & 7.62 & 9.43 & 9.54 & 51.30 & - \\
\hline
\end{tabular}

The values that indicate dissimilarity are italicised



Fig. 3 WAXS diffractograms: a nimodipine (NM), mesoporous silica (MCM), physical mixture (NM-M), silica with encapsulated NM: unwashed sample (NM-M-1) and washed sample (NM-M-2); b nimodipine (NM), mesoporous silica (SBA), physical mixture (NM-S), silica with encapsulated NM: unwashed sample (NM-S-1) and washed sample (NM-S-2) presented in the literature. Here, we would like to fill the void by presenting the band assignment, supported by the state-of-the art first-principles calculations, dedicated to solid-state. As mentioned above, NM can form two polymorphic forms, which can coexist under ambient conditions. In the present paper, we refer the analysis only to the monoclinic polymorph, because-as shown by the WAXS measurements (cf. Powder X-ray diffractionWAXS) - the sample studied here can be classified as NM form I.

The optical vibrational spectra of both polymorphic forms have been reported by Grunenberg et al. [3] and recently extensively evaluated with chemometry by Siddiqui et al. [44]. The experimental FT-IR spectrum of our sample stays in excellent agreement with the data reported in literature for NM form I.
The detailed band assignment of NM form I is given in Table 2 (on the basis of DFT calculations), while a comparison of experimental and theoretical spectra-calculated for both isolated molecule and solid-state models-is presented in Fig. 4.

Because of the $\mathrm{P} 2{ }_{1} / \mathrm{c}$ symmetry and the presence of 4 molecular moieties in the unit cell, the 669 optical-active modes are distributed among the symmetry species as follows: $\Gamma=166 \mathrm{~B}_{u}+167 \mathrm{~A}_{u}+168 \mathrm{~B}_{g}+168 \mathrm{~A}_{g}$. Hence, 335 ungerade symmetry modes are expected in the solidstate FT-IR spectrum, in which the wavenumbers of modes are further affected by the factor-group splitting. The calculated spectra are in very good agreement with the experimental data, allowing for a reliable band assignment.

The highest-wavenumber range exhibits the characteristic $v(\mathrm{~N}-\mathrm{H})$ stretching band, which is affected by the 
Fig. 4 The FT-IR spectrum of $\mathrm{NM}$ form I, against the results of the theoretical DFT calculations, performed in frame of isolated molecule (DMOL3/rPBE/TNP) and solidstate (CASTEP/rPBE/1050 eV) models. Note the slight wavenumber scale for the theoretical results $\left(<1750 \mathrm{~cm}^{-1}\right)$, introduced to account the systematic errors in DFT calculations



3600330030002700
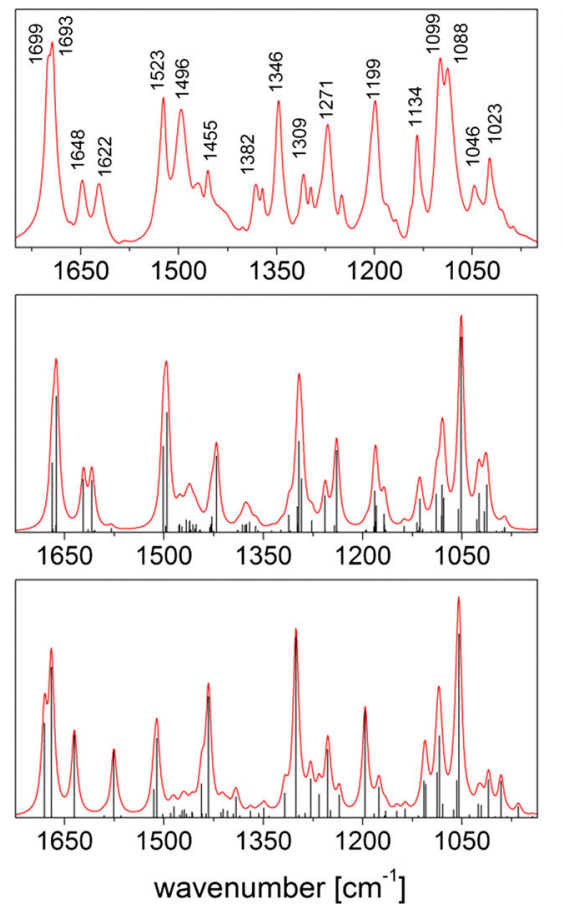
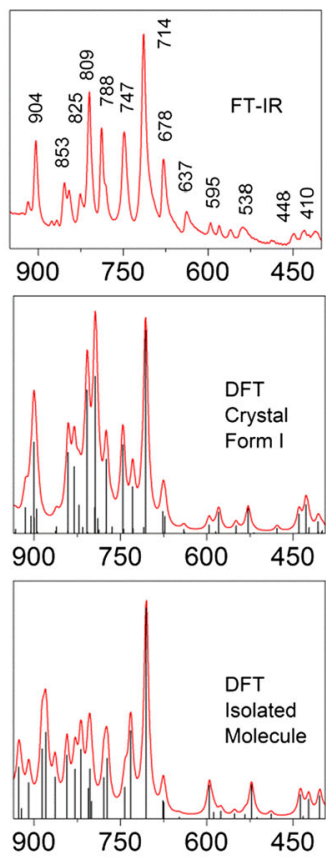

hydrogen-bonding — as one may clearly note by comparing molecular and crystal-phase calculations. Below this region, one may note the $\mathrm{v}(\mathrm{C}-\mathrm{H})$ stretchings associated with the aromatic ring (3221 and $3097 \mathrm{~cm}^{-1}$ ) and terminal chains $\left(2984,2935\right.$ and $\left.2822 \mathrm{~cm}^{-1}\right)$.

The most intense, double band with the peaks at 1699 and $1693 \mathrm{~cm}^{-1}$ is assigned to the carbonyl stretching modes, slightly mixed the $\delta(\mathrm{C}-\mathrm{N}-\mathrm{H})$ bendings. The two characteristic bands at 1648 and $1622 \mathrm{~cm}^{-1}$ are dominated by the $v(\mathrm{C}=\mathrm{C})$ stretchings in dihydropyridine ring with some contributions from the $v(\mathrm{C}=\mathrm{O})$ and $\delta(\mathrm{C}-\mathrm{N}-\mathrm{H})$ modes. By comparing the molecular and crystal-phase calculations, it is clear that the formation of $\mathrm{N}-\mathrm{H} \cdots \mathrm{O}$ bond reduces their separation.

The characteristic intense band, assigned to the antisymmetric $v \mathrm{NO}_{2}$ stretching, was found at $1523 \mathrm{~cm}^{-1}$ (it symmetric counterpart was observed at $1346 \mathrm{~cm}^{-1}$ ). The mode at $1496 \mathrm{~cm}^{-1}$ refers to the $\delta(\mathrm{C}-\mathrm{N}-\mathrm{H})$ bendings. The remaining bands in the range $1500-1450 \mathrm{~cm}^{-1}$ correspond to the antisymmetric $\delta\left(\mathrm{CH}_{3}\right)$ and $\delta\left(\mathrm{CH}_{2}\right)$ bendings, while the symmetric $\delta\left(\mathrm{CH}_{3}\right)$ umbrella modes are observed down to $1350 \mathrm{~cm}^{-1}$. The in-plane $\delta(\mathrm{C}-\mathrm{C}-\mathrm{H})$ bendings within the aromatic part are spread over the range $1300-1100 \mathrm{~cm}^{-1}$.

The strong bands found at 1134 and $1099 \mathrm{~cm}^{-1}$ can be linked to the $v(\mathrm{C}-\mathrm{O})$ stretching in the ether and ester groups, mixed with the $v\left(\mathrm{C}^{*}-\mathrm{C}\right)$ bond stretching in the dihydropyridine ring. Beneath, the phenyl ring trigonal mode is observed as the very intense band at $1088 \mathrm{~cm}^{-1}$. The $v(\mathrm{C}-\mathrm{O})$ stretching modes also contribute to the medium intensity bands observed at 1023, 904 and $853 \mathrm{~cm}^{-1}$, respectively.
The range of $\sim 825-550 \mathrm{~cm}^{-1}$, restored with excellent accuracy by the solid-state calculations, is dominated by the out-of-plane vibrations. While the $\gamma(\mathrm{CH})$ modes dominate the spectrum down to $\sim 700 \mathrm{~cm}^{-1}$, the lower part of the spectrum exposes the out-of-plane ring deformations.

The lowest analysed part of the FT-IR spectrum is generally mainly covered by the weak-intensity bands, linked to the complex skeletal deformations of the terminal chains, which becomes more delocalized when going down with the wavenumbers.

\subsubsection{Vibrational analysis of encapsulated samples}

The initial materials i.e. NM and mesoporous matrices as well as products of their encapsulation and washing were also characterised by FT-IR spectra (Fig. 5).

The FT-IR spectra of silica matrices MCM-41 and SBA15 are similar because of the alike composition of these two materials. They both show e.g. a narrow band assigned to the stretching vibrations of surface hydroxyl groups of single and geminal type (well seen at $3746 \mathrm{~cm}^{-1}$ in the spectrum of MCM-41 and poorly seen at $3747 \mathrm{~cm}^{-1}$ in that of SBA-15). There is also a broad band assigned to the stretching vibrations of hydrogen- bonded surface hydroxyl groups (at $3420 \mathrm{~cm}^{-1}$ in the spectrum of MCM-41 and at $3446 \mathrm{~cm}^{-1}$ in that of SBA-15) [45]. Other bands include: that assigned to bending vibrations $\delta \mathrm{H}-\mathrm{O}-\mathrm{H}$ of adsorbed water (at $1631 \mathrm{~cm}^{-1}$ in the spectrum of MCM-41 and $1635 \mathrm{~cm}^{-1}$ in that of SBA-15), a broad band assigned to bending vibrations $\delta \mathrm{Si}-\mathrm{O}-\mathrm{Si}$ (at $1089 \mathrm{~cm}^{-1}$ in the spectrum of MCM-41 and $1082 \mathrm{~cm}^{-1}$ in that of SBA-15). 
Fig. 5 FT-IR spectra a nimodipine (NM), mesoporous silica (MCM), physical mixture (NM-M) and samples with encapsulated nimodipine: unwashed (NM-M1) and washed (NM-M-2); b nimodipine (NM), mesoporous silica (SBA), physical mixture (NM-S) and samples with encapsulated nimodipine: unwashed (NM-S1) and washed (NM-S-2)

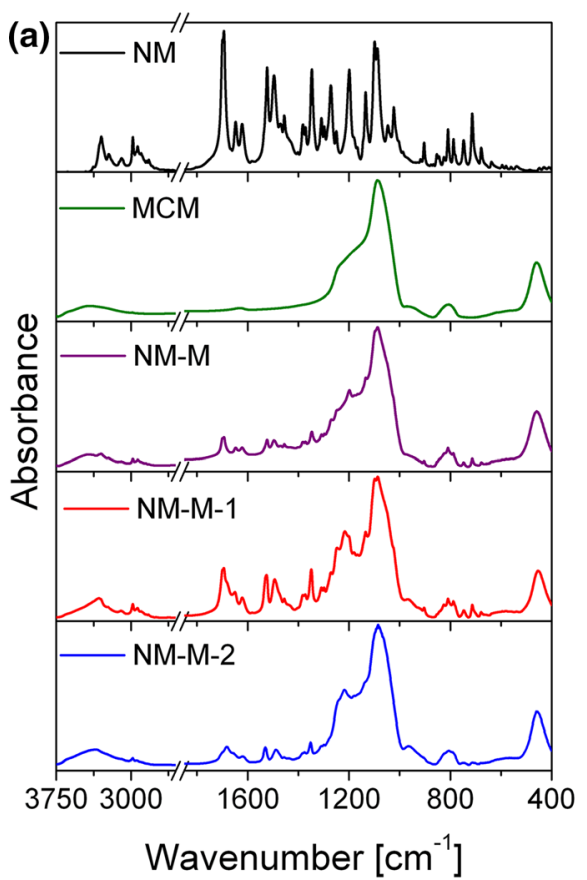

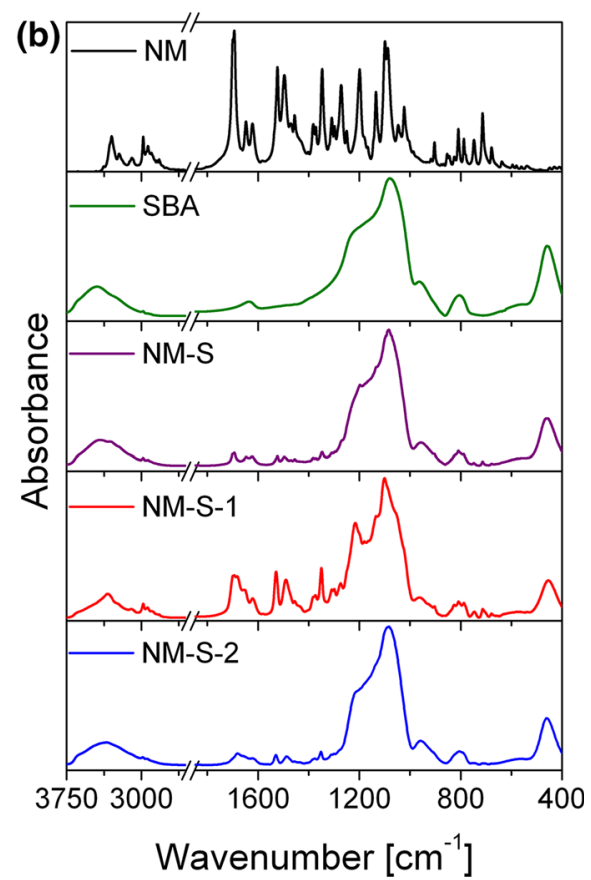

Below $1000 \mathrm{~cm}^{-1}$, there is also a band assigned to out-ofplane vibrations $\gamma \mathrm{H}_{\text {silanol }} \cdots \mathrm{O}-\mathrm{H}_{\text {water }}$ (at $806 \mathrm{~cm}^{-1}$ in the spectrum of MCM-41 and at $805 \mathrm{~cm}^{-1}$ in that of SBA-15) and a band assigned to $\delta \mathrm{O}-\mathrm{Si}-\mathrm{O}$ and $\delta \mathrm{Si}-\mathrm{O}-\mathrm{Si}$ bending vibrations (at $460 \mathrm{~cm}^{-1}$ in the spectrum of MCM-41 and at $458 \mathrm{~cm}^{-1}$ in that of SBA-15). As suggested in literature, in this region a band assigned to libration modes of water should be observed, but its absence in our samples may be a result of quite low water content [46, 47].

As follows from Fig. 5, the FT-IR spectra of physical mixtures (NM-M/NM-S) are the superpositions of the spectrum of NM and the appropriate silica.

However, in the spectra of silica with encapsulated NM (NM-M-1/NM-M-2 and NM-S-1/NM-S-2) some differences may be noted with respect to those of physical mixture (NM-M/NM-S). For the samples supported on MCM-41 (NM-M-1/NM-M-2) the process of NM encapsulation results in a disappearance of the band assigned to the stretching vibrations of the surface $\mathrm{O}-\mathrm{H}$ of single and geminal types $\left(3746 \mathrm{~cm}^{-1}\right)$. It means that encapsulation of $\mathrm{NM}$ in the silica matrix leads to a reduction of the number of unbounded silanol groups. Thus, it can be assumed that - through formation of hydrogen bonds-these groups are involved in the immobilisation of NM on the surface of silica pores. Similar effect was observed in the spectra of samples supported on SBA-15 (NM-S-1/NM-S-2), however for this matrix the disappearance of the band at $3747 \mathrm{~cm}^{-1}$ is much less noticeable.

The most pronounced changes in the spectra of the samples after encapsulation are observed near $\sim 1700 \mathrm{~cm}^{-1}$ (region of $\mathrm{vC}=\mathrm{O}$ vibrations) and near $\sim 3300 \mathrm{~cm}^{-1}$ (region of $v \mathrm{~N}-\mathrm{H}$ vibrations). In the first region, i.a. near $\sim 1700 \mathrm{~cm}^{-1}$, besides the band at $1693 \mathrm{~cm}^{-1}$ (characteristic for $\mathrm{vC}=\mathrm{O}$ vibrations of crystalline NM), another band appeared at a distance of nearly $10 \mathrm{~cm}^{-1}$. In the spectra of samples supported on MCM-41 it was localised at $1700 \mathrm{~cm}^{-1}$, while in those of the samples supported on SBA-15-at $1683 \mathrm{~cm}^{-1}$. This splitting can be attributed to the presence of the amorphous form of NM which appears as a result of encapsulation [48, 49]. Moreover, it was observed that the relative content of the amorphous form of NM increases after washing, as the ratio of intensity of the new band (at 1700 and $1683 \mathrm{~cm}^{-1}$ ) to that of the band assigned to $\mathrm{vC}=\mathrm{O}$ vibration of crystalline form of NM $\left(1693 \mathrm{~cm}^{-1}\right)$ was much higher for the washed sample. It can be assumed that the effect observed is a result of removal of the NM crystallites from the external surface of a sample (cf. Powder X-ray diffractionWAXS).

In the second region, i.a. near $\sim 3300 \mathrm{~cm}^{-1}$, in the spectra of both matrices with encapsulated NM, for unwashed as well as washed samples, there is no band observed that is assigned to the $\mathrm{vN}-\mathrm{H}$ vibrations of $\mathrm{NM}$ in crystalline form $\left(3296 \mathrm{~cm}^{-1}\right)$. Its disappearance may suggest the involvement of this group in formation of hydrogen bonds with the silanol groups [50], which indicates a potential mechanism of NM bonding to the silica matrix during encapsulation. Additional evidence of the presence of amorphous NM is the appearance of the bands at $3323 \mathrm{~cm}^{-1}$ in the spectra of samples supported on MCM- 
41 and at $3338 \mathrm{~cm}^{-1}$ in the spectra of samples supported on SBA-15, especially well seen in the spectra of washed samples (NM-M-2/NM-S-2).

From the analysis of the FT-IR spectra it can be concluded that during the process of encapsulation additional hydrogen bonds arise in the system. Most probably they are formed between the amino group of NM and oxygen atoms of the silanol groups from the matrix. Furthermore, the additional bands appearing at 1700 and $3323 \mathrm{~cm}^{-1}$ for samples supported on MCM-41 and at 1683 and $3338 \mathrm{~cm}^{-1}$ for samples supported on SBA-15, are related to the presence of amorphous form of NM.

\subsection{Scanning and transmission electron microscopies}

The size of particles and surface morphology of the mesoporous silicas MCM-41 and SBA-15 were analysed on the basis of SEM images, presented in Fig. 6. In the micrometric scale the materials are very similar. As can be seen form Fig. 6, for both samples the SEM images show aggregations of grains, whose sizes are approximately from more than ten (SBA) to a few tens (MCM) of micrometers, forming a rich variation of surface structures. In the system of so rich morphology it is not possible to perform encapsulation of a drug without its considerable adsorption on the external surface of the material. Thus, the rich morphology explains the presence of NM crystallites in unwashed samples (cf. Thermal analysis-DSC and Powder X-ray diffraction-WAXS) and emphasise the significance of washing procedure in the preparation of the final product (cf. Drug loading).

Despite being similar in a SEM recording scale, the two silica matrices: MCM-41 and SBA-15 revealed a significant difference in morphology in TEM images (Fig. 7). Although the materials exhibit highly ordered, hexagonal porous structure, in MCM-41 the domains (of the sizes near $50 \mathrm{~nm}$ ) are distributed randomly, while in SBA-15 they assume the shape of regular tubes (of about $0.5 \mu \mathrm{m}$ in diameter and over $10 \mu \mathrm{m}$ in length). Such morphology, especially that of SBA-15, limits the accessibility of pores and thus also the sorption ability of SBA- 15 with respect to that of MCM-41. However, no such differences were noted when comparing their surface areas, as they reached very close values for both silicas. Thus, unlike the nitrogen sorption results (cf. Basic characterisation), the differences
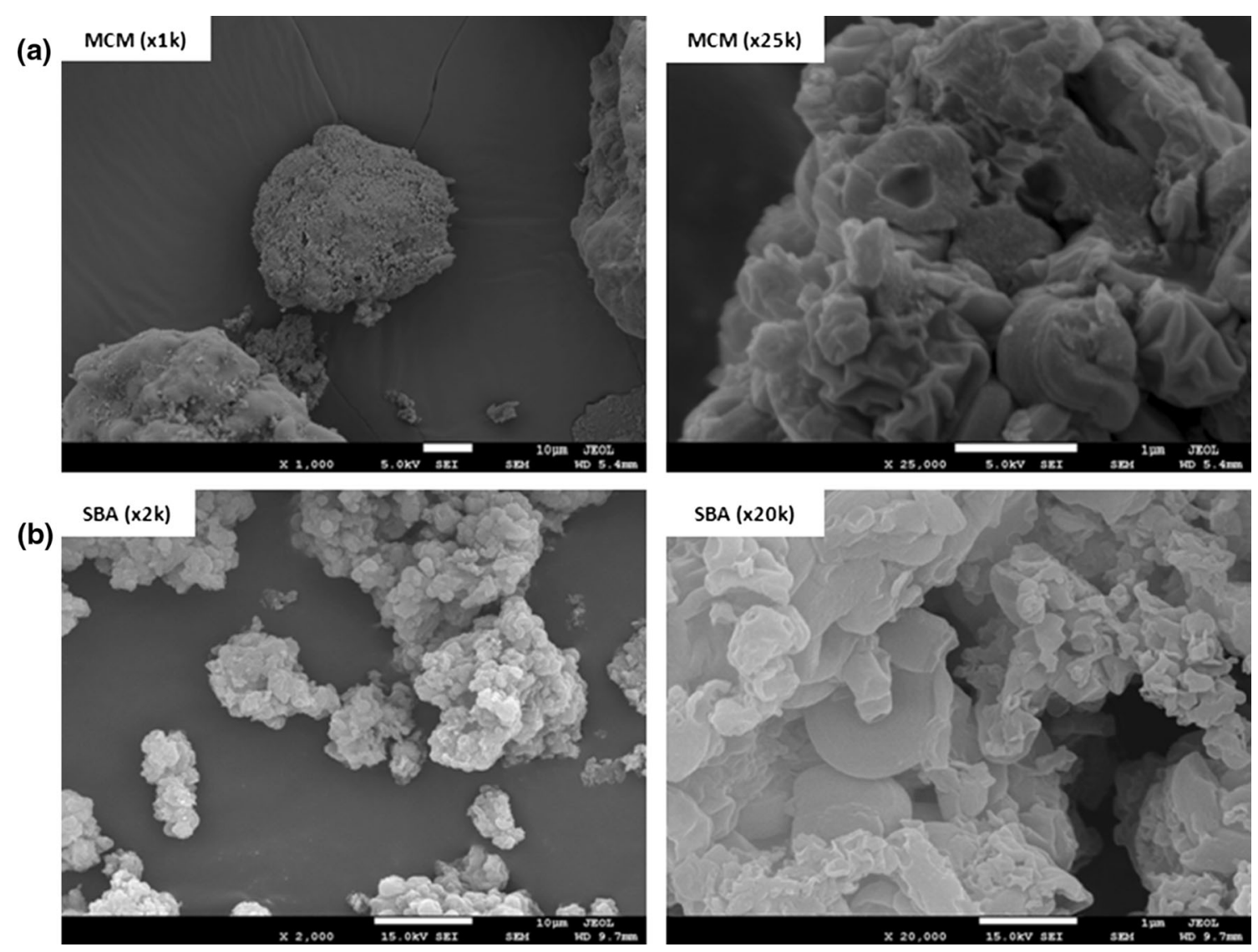

Fig. 6 SEM images of the silica matrices used for the encapsulation a MCM-41-magnification $\times 1000($ left $)$ and $\times 25,000($ right $)$; b SBA-15magnification $\times 2000($ left $)$ and $\times 20,000($ right $)$ 
Fig. 7 TEM images of a MCM-41, b SBA-15, before (left) and after (right) NM encapsulation and washing procedure
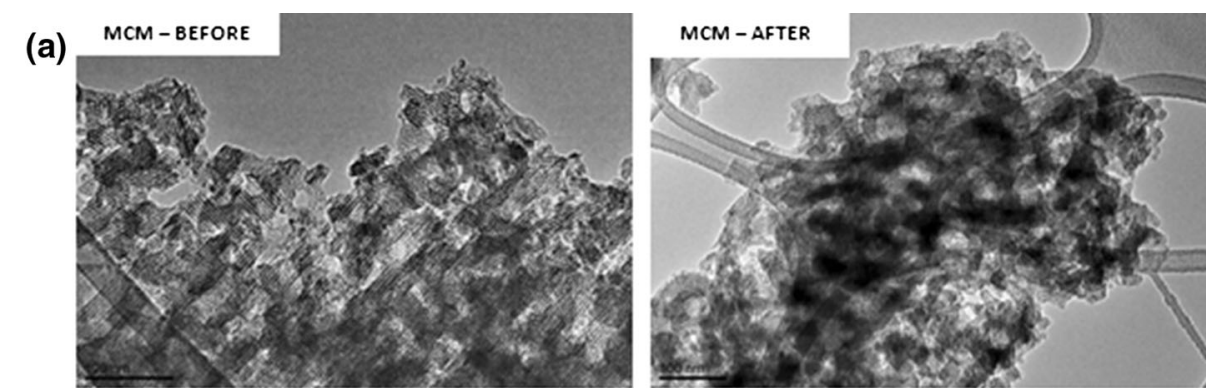

(b)


revealed by TEM method have explained why - as a result of the same process of NM encapsulation-a slightly lower amount of NM was incorporated in SBA-15 than in MCM41 (cf. TGA).

Moreover, as shown in the TEM images (Fig. 7, the process of encapsulation does not affect the morphology of silica matrices and does not degrade the porous structure, as it has been also quantitatively implied by SAXS measurements (cf. SAXS).

\subsection{Drug dissolution and release studies}

An aqueous equilibrium solubility of NM was reported to be $3.86 \mathrm{mg} / \mathrm{L}$ [51]. Our dissolution and release studies of NM have been performed under supersaturating conditions using $1 \mathrm{~L}$ of ultra pure water and appropriated amounts of powders corresponding to $5 \mathrm{mg}$ of NM. Non-sink conditions were chosen, as drug loaded mesoporous silica materials might lead to a temporary supersaturation of the dissolution medium. Therefore, under non-sink conditions a better differentiation of NM loaded silica carriers and comparison of the degree and duration of supersaturation reached is possible. Figure 8 shows the dissolution and release profiles of NM in ultra pure water.
Comparison of NM dissolution and release profiles from all studied samples was performed by similarity factor $f_{2}$ method (Equation (1) - supporting materials) and the values obtained are summarized in Table 2. As seen in Table 2, $f_{2}$ values for NM-M-2 or NM-S-2 versus NM, NM-M, NM-S, NM-M-1 and NM-S-1 indicate lack of profiles similarity $\left(f_{2}<50\right)$. Release profiles of NM loaded in MCM-41 and washed (NM-M-2) show comparative results with NM-S-2 $\left(f_{2}=51.3\right)$. We also observed dissimilarity between the dissolution curves of NM-M-1 or NM-S-1 versus NM.

The influx and competitive adsorption of water to the pore surfaces provides for a rapid dissolution and complete release of NM from NM-S-2 and NM-M-2 materials. The drug loaded in these mesoporous materials show very fast initial drug release profiles: more than $60 \%$ of the incorporated nimodipine was dissolved and released within $3 \mathrm{~min}$. On the other hand we observed very poor and incomplete dissolution of nimodipine (32.80-41.35\% at $720 \mathrm{~min}$ ) from all other samples containing crystalline form: NM, NM-M, NM-S, NM-M-1 and NM-S-1. The maximum fraction $\left(\mathrm{F}_{\max }\right)$ of the drug in solution was extracted directly from the experimental data and for NM-M$2 \mathrm{~F}_{\max }$ was equal to $102.95 \pm 4.28 \%$ at $165 \mathrm{~min}$ (see 

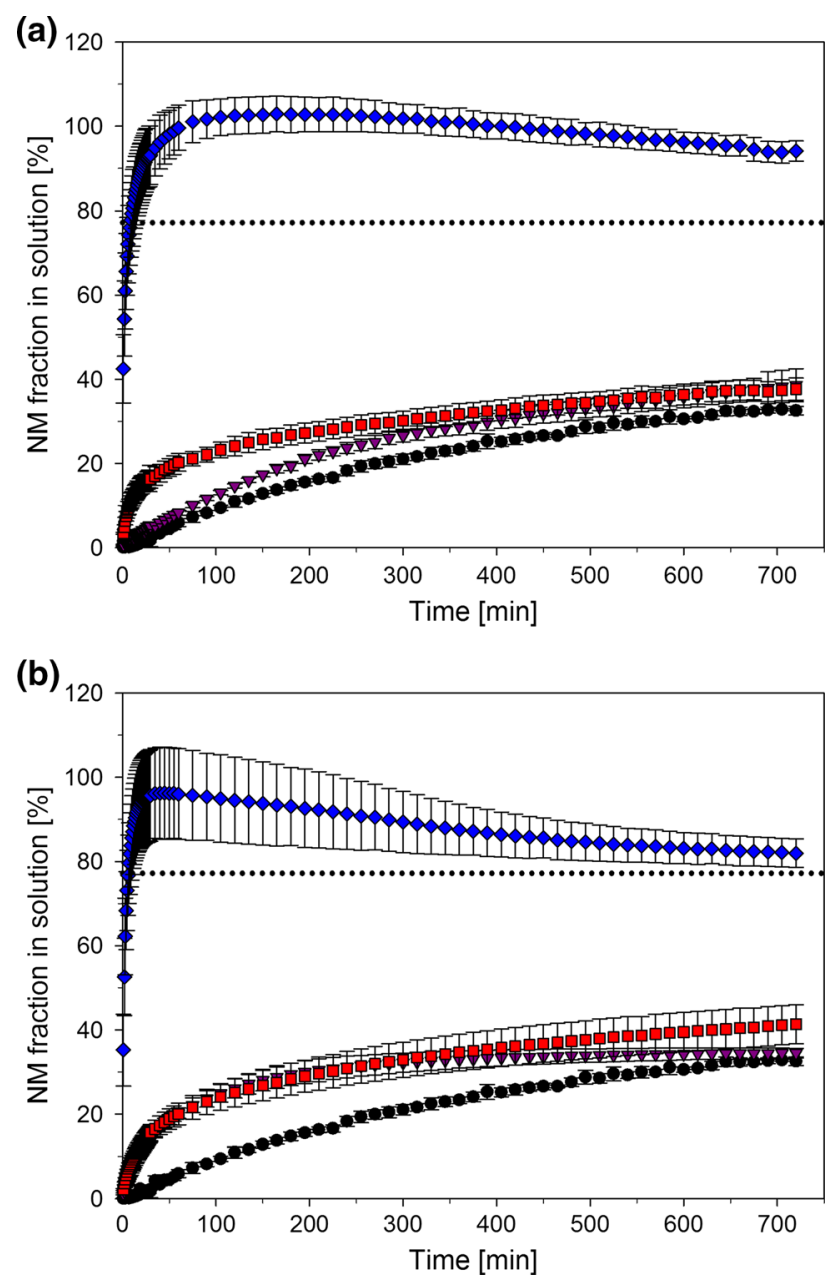

Fig. 8 Dissolution and release profiles of powders containing $5 \mathrm{mg}$ of $\mathrm{NM}$ (calculated as an average from $\mathrm{n}=3$ experiments) in $1 \mathrm{~L}$ of ultra pure water: a nimodipine (NM) - black circles, physical mixture (NM-M) - purple triangles, silica with encapsulated NM: unwashed sample (NM-M-1)—red squares and washed sample (NM-M-2) blue lozenges; $\mathbf{b}$ nimodipine (NM) - black circles, physical mixture (NM-S)_purple triangles, silica with encapsulated NM: unwashed sample (NM-S-1) — red squares and washed sample (NM-S-2) — blue lozenges. The dotted line represent thermodynamic solubility of nimodipine in ultra pure water $\left(\mathrm{C}_{\mathrm{s}}=3.86 \mathrm{mg} / \mathrm{L}\right)$ [51]

Fig. 8a), while for NM-S-2 was $96.24 \pm 10.93 \%$ at $45 \mathrm{~min}$ (see Fig. 8b). The first-order model (Equation (2) - supporting materials) was fitted to three individual dissolution data of each sample of NM. This model provides a good fit for all of the dissolution and release profiles $\left(\mathrm{R}_{\text {observed-predicted }}>0.95\right)$, indicating similar Fickian diffusion mechanism of NM release from tested materials $[52,53]$. Release rate of NM from NM-S-2 with $\mathrm{k}_{\mathrm{D}}=0.3792\left(\mathrm{~min}^{-1}\right)$ was 108 times faster than dissolution rate of bulk $\mathrm{NM}\left[\mathrm{k}_{\mathrm{D}}=0.0035\left(\mathrm{~min}^{-1}\right)\right]$. Release rate of NM from NM-M-2 $\left[k_{\mathrm{D}}=0.2485\left(\mathrm{~min}^{-1}\right)\right]$ was approximately 70 times faster than dissolution rate of bulk NM. Compared with the crystalline drug, the amorphous phase of nimodipine inside the mesopores can reduce the lattice energy and improve the wettability, resulting in enhanced dissolution and release behaviour [54]. The 1.5 times faster delivery of NM from NM-S-2 than from NMM-2 could be related to the difference of the pore size between SBA-15 and MCM-41 (1.7 times smaller pores in MCM-41) [55].

However, the NM amounts released within first 2 min were higher from NM-M-2 (42.48\% at $1 \mathrm{~min}$ and $54.39 \%$ at $2 \mathrm{~min})$ with the smaller pore size $(3.66 \mathrm{~nm})$ than from NM-S-2 (35.20\% at $1 \mathrm{~min}$ and $52.58 \%$ at $2 \mathrm{~min})$. These results demonstrate the effect of the particle morphology on drug release. TEM images revealed significant differences in sizes and shapes of MCM-41 and SBA-15 matrices (Fig. 7) which could be ascribed to the difference in the length of pore channels [56]. Particles having short pore channels (i.e. spherical MCM-41 with diameter ca. $0.05 \mu \mathrm{m}$ ) could further reduce diffusion resistance for NM release to the dissolution media. Cylindrical SBA-15 with dimensions $0.5 \times 10 \mu \mathrm{m}$ has longer diffusional channels that the molecules have to follow to reach the bulk solution.

Furthermore, it was shown that the burst-like release of the amorphous NM from ordered mesoporous silica materials gave rise to supersaturation in ultra pure water which caused only limited precipitation (entropy-driven crystallization). The more gradual release of NM from MCM-41 results in a more sustained supersaturation (Fig. 8a) compared with SBA-15 (Fig. 8b). To assess the stability of supersaturation of the respective formulation, the corrected area under the concentration-time profile (cAUC) was calculated. It should be noted that the area that corresponds to the thermodynamic solubility of nimodipine in ultra pure water (i.e., the area under the dotted line in Fig. 8) was subtracted from the area under the curve of the NM-M-2 and NM-S-2. Thus the cAUC represents a measure of supersaturation, taking into account both the degree and the duration of supersaturation [57]. The cAUC increases progressively with decreasing release rate (influenced by pore size, amount of silanol groups per surface area, drug loading, pore filling and particle morphology): 15546 (\% $\mathrm{min})$ and 7878 (\% $\mathrm{min}$ ) for NM-M-2 and NM-S2 , respectively. The maximum degree of supersaturation (i.e., the highest concentration attained, $\mathrm{F}_{\max }$ ) is slightly higher for NM-M-2. Moreover, the time point of maximal supersaturation is 3.7 times increased for NM-M-2 compared to NM-S-2. Supersaturated concentrations were maintained for at least $11 \mathrm{~h}$ from both matrices (see Fig. 8). According to first-order model (Equation (3)supporting materials) less precipitation was observed for NM-M-2 with precipitation rate $\mathrm{k}_{\mathrm{P}}=0.00017\left(\mathrm{~min}^{-1}\right)$ which is 1.5 times smaller than for NM-S-2 $\left[\mathrm{k}_{\mathrm{P}}=0.00026\right.$ $\left.\left(\min ^{-1}\right)\right]$. 
These results indicate the improvement of NM dissolution and release rate due to the following factors: amorphization, increase its specific surface area using mesoporous materials and the weak interactions between silica surface and the drug which was easily removed in presence of water. These rapid kinetic profiles lead to supersaturation state and present great interest for pharmaceutical application in order to enhance dissolution and improve a rapid drug delivery of poorly water-soluble drugs.

\section{Conclusion}

Two hexagonally ordered mesoporous silica materials (MCM-41 and SBA-15) were applied as potential nanocarriers for poorly soluble drug-nimodipine. Nimodipine was encapsulated using method of modified adsorption and obtained samples were studied before and after washing. As the results of TGA analysis have shown, this type of adsorption for both mesoporous matrices gave the drug loading value of approximately $50 \%$ before and $20 \%$ after washing procedure.

According to DSC, WAXS and FT-IR results, nimodipine appeared in two different states: the crystalline one, that was easily removed during washing, and the amorphous one, that allowed to improve some physicochemical properties of studied material i.e. the release rate. The presence of amorphous nimodipine as well as the hydrogen bonding between its molecules and the surface of silica matrix were detected using FT-IR analysis, interpreted partly on the basis of first-principles calculations.

Dissolution and drug release experiments in ultra pure water in non-sink conditions showed fast and complete release of drug molecules included within SBA-15 and MCM-41 mesopores. Supersaturation was observed for amorphous formulations and sustained for at least $11 \mathrm{~h}$. Significant improvement of drug dissolution and release was observed as compared to the dissolution of the pure drug of crystalline phase or to its physical mixtures' with SBA-15 or MCM-41 and drug crystals as well as to unwashed mesoporous materials.

Therefore, the results evidenced that SBA-15 and MCM-41 are a promising carriers to achieve amorphous form and enhanced dissolution rate for nonionisable, poorly water-soluble drug-nimodipine.

Acknowledgments This work has been partially financed by the EU Operational Programme 'Human Capital' (PO KL 4.1.1), the National Science Centre of Poland, Grant No. 2012/05/B/ST3/03176 and Poznan University of Medical Sciences Grant No. 502-01-033-14429-03439. K. Drużbicki acknowledges the financial support of Polish Government Plenipotentiary for JINR in Dubna (Grant No. 118-8/ 1069-5/2014). This research was also supported in part by PL-Grid
Infrastructure (Grant ID: postdoc, phd2013). M. Grzeszkowiak acknowledges the support of the European Social Fund within the project POKL.04.03.00-00-015/12. Financial support from the National Centre for Research and Development under research grant "Nanomaterials and their application to biomedicine" (contract number PBS1/A9/13/2012) is also gratefully acknowledged. We also thank I. Mizera and K. Hyla for their assistance with the dissolution and drug release experiments.

Open Access This article is distributed under the terms of the Creative Commons Attribution License which permits any use, distribution, and reproduction in any medium, provided the original author(s) and the source are credited.

\section{References}

1. D.D. Freedman, D.D. Waters, Drugs 34, 578-598 (1987)

2. K.E. Thummel, K.L. Kunze, D.D. Shen, Adv. Drug Deliv. Rev. 27, 99-127 (1997)

3. A. Grunenberg, B. Keil, J.O. Henck, Int. J. Pharm. 118, 11-21 (1995)

4. M.K. Riekes, R.N. Pereira, G.S. Rauber, S.L. Cuffini, C.A. Maduro de Campos, M.A.S. Silva, H.K. Stulzera, J. Pharm. Biomed. Anal. 70, 188-193 (2012)

5. Y. Kawabata, K. Wada, M. Nakatani, S. Yamada, S. Onoue, Int. J. Pharm. 420, 1-10 (2011)

6. E. Vinge, K.E. Andersson, L. Brandt, B. Ljunggren, L.G. Nilsson, S. Rosendal-Helgesen, Eur. J. Clin. Pharmacol. 30, 421-425 (1986)

7. G.M. Soliman, R. Sharma, A.O. Choi, S.K. Varshney, F.M. Winnik, A.K. Kakkar, D. Maysinger, Biomaterials 31, 8382-8392 (2010)

8. S.C. Yang, H.X. Ge, Y. Hu, X.Q. Jiang, C.Z. Yang, Colloid Polym. Sci. 278, 285-292 (2000)

9. S. Papadimitriou, G.Z. Papageorgiou, F.I. Kanaze, M. Georgarakis, D.N. Bikiaris, J. Nanomater. (2009) Article ID 716242. doi: $10.1155 / 2009 / 716242$

10. H. Ge, Y. Hu, S.C. Yang, X.Q. Jiang, C.Z. Yang, J. Appl. Polym. Sci. 75, 874-882 (2000)

11. H. Ge, Y. Hu, X.Q. Jiang, D. Cheng, Y. Yuan, H. Bi, C.Z. Yang, J. Pharm. Sci. 91, 1463-1473 (2002)

12. Y. Hu, X.Q. Jiang, Y. Ding, L. Zhang, C.Z. Yang, J. Zhang, J. Chen, Y. Jang, Biomaterials 24, 2395-2404 (2003)

13. X.H. Dai, H.D. Zhang, ChM Dong, Polymer 50, 4626-4634 (2009)

14. M.K. Riekes, L.R.O. Luiz, R.N. Pereira, P.A.A. Borba, D. Fernandes, H.K. Stulzer, Curr. Pharm. Des. 19, 7264-7270 (2013)

15. A.K. Mehta, K.S. Yadav, K.K. Sawant, Curr. Drug Deliv. 4, 185-193 (2007)

16. Q. Liu, C. Cai, C. Dong, J. Biomed. Mater. Res. Part A 88, 990-999 (2009)

17. N. Bege, T. Renette, T. Endres, M. Beck-Broichsitter, D. Hänggi, T. Kissel, Eur. J. Pharm. Biopharm. 84, 99-105 (2013)

18. H.S. Abyaneh, M.R. Vakili, A. Lavasanifar, Pharm. Res. 31, 1485-1500 (2014)

19. Q. Zhang, X. Jiang, W. Jiang, W. Lu, L. Su, Z. Shi, Int. J. Pharm. 275, 85-96 (2004)

20. R. Xiong, W. Lu, J. Li, P. Wang, R. Xu, T. Chen, Int. J. Pharm. 350, 338-343 (2008)

21. N.A. Urbanetz, BCh. Lippold, Eur. J. Pharm. Biopharm. 59, 107-118 (2005)

22. N.A. Urbanetz, Eur. J. Pharm. Sci. 28, 67-76 (2006)

23. G.Z. Papageorgiou, A. Docoslis, M. Georgarakis, D. Bikiaris, J. Therm. Anal. Calorim. 95, 903-915 (2009) 
24. A. Yoshida, M. Yamamoto, T. Itoh, T. Irie, F. Hirayama, K. Uekama, Chem. Pharm. Bull. 38, 176-179 (1990)

25. Y.Z. Du, J.G. Xu, L. Wang, H. Yuan, F.Q. Hu, Eur. Polym. J. 45, 1397-1402 (2009)

26. S.K. Majahar, R.M. Kusumanchi, B.N. Gayatri, Int. J. Pharm. Biol. Sci. 1, 1-8 (2010)

27. J. Yu, H.B. He, X. Tang, J. Pharm. Pharmacol. 58, 1429-1435 (2006)

28. F.Q. Hu, Y. Zhang, Y.Z. Du, H. Yuan, Int. J. Pharm. 348, 146-152 (2008)

29. S.S. Chalikwar, V.S. Belgamwar, V.R. Talele, S.J. Surana, M.U. Patil, Colloids Surf. B 97, 109-116 (2012)

30. D. Yang, J. Zhu, Y. Zheng, L. Ge, Drug Dev. Ind. Pharm. 32, 219-227 (2006)

31. X. Song, Y. Jiang, C. Ren, X. Sun, Q. Zhang, T. Gong, Z. Zhang, Int. J. Nanomedicine 7, 3689-3699 (2012)

32. E.S. Gil, S.M. Hudson, Prog. Polym. Sci. 29, 1173-1222 (2004)

33. Q. Fu, J. Sun, D. Zhang, M. Li, Y. Wang, G. Ling, X. Liu, Y. Sun, X. Sui, C. Luo, L. Sun, X. Han, H. Lian, M. Zhu, S. Wang, Z. He, Colloid Surf. B 109, 161-166 (2013)

34. S. Wang, Microporous Mesoporous Mater. 117, 1-9 (2009)

35. H. Yu, Q.Z. Zhai, Microporous Mesoporous Mater. 123, 298-305 (2009)

36. Z.G. Shi, Q.Z. Guo, Y.T. Liu, Y.X. Xiao, L. Xu, Mater. Chem. Phys. 126, 826-831 (2011)

37. D. Zhao, J. Feng, Q. Huo, N. Melosh, G.H. Fredrickson, B.F. Chmelka, G.D. Stucky, Science 279, 548-552 (1998)

38. D. Zhao, Q. Huo, J. Feng, B.F. Chmelka, G.D. Stucky, J. Am. Chem. Soc. 120, 6024-6036 (1998)

39. European Pharmacopoeia Commission, Council of Europe, European Pharmacopoeia, 8th edn. (European Directorate for the Quality of Medicines \& Health Care, Strasbourg, 2013)

40. S. Ek, A. Root, M. Peussa, L. Niinistö, Thermochim. Acta 379, 201-212 (2001)
41. S.D. Wang, L.G. Herbette, D.G. Rhodes, Acta Crystallogr. C 45, 1748-1751 (1989)

42. D.A. Langs, P.D. Strong, D.J. Triggle, J. Comput. Aided Mol. Des. 4, 215-230 (1990)

43. Ch. Huo, J. Ouyang, H. Yang, Sci. Rep. 4, 3682 (2014)

44. A. Siddiqui, Z. Rahman, V.A. Sayeed, M.A. Khan, J. Pharm. Sci. 102, 4024-4035 (2013)

45. X.S. Zhao, Q. Lu, A.K. Whittaker, G.J. Millar, H.Y. Zhu, J. Phys. Chem. B 101, 6525-6531 (1997)

46. X. Wang, P. Wang, Z. Dong, Z. Ma, J. Jiang, R. Li, J. Ma, Nanoscale Res. Lett. 5, 1468-1473 (2010)

47. P.K. Gupta, M. Meuwly, Faraday Discuss. 167, 329-346 (2013)

48. XCh. Tang, M.J. Pikal, L.S. Taylor, Pharm. Res. 19, 477-483 (2002)

49. P. Barmpalexis, K. Kachrimanis, E. Georgarakis, Eur. J. Pharm. Biopharm. 77, 122-131 (2011)

50. X. Zheng, R. Yang, X. Tang, L. Zheng, Drug Dev. Ind. Pharm. 33, 791-802 (2007)

51. Y.Z. Sun, R. Yang, L.W. Zhou, X. Tang, Int. J. Pharm. 359, 144-149 (2008)

52. P. Costa, J.M. Sousa Lobo, Eur. J. Pharm. Sci. 13, 123-133 (2001)

53. FDA, Guidance for Industry: Dissolution Testing of Immediate Release Solid Oral Dosage Forms (Rockville, 1997)

54. W. Hu, J. Riikonen, V.P. Lehto, Int. J. Pharm. 453, 181-197 (2013)

55. P. Horcajada, A. Ramila, J. Perez-Pariente, M. Vallet-Regi, Microporous Mesoporous Mater. 68, 105-109 (2004)

56. F. Qu, G. Zhu, S. Huang, S. Li, J. Sun, D. Zhang, S. Qiu, Microporous Mesoporous Mater. 92, 1-9 (2006)

57. M. Van Speybroeck, R. Mellaerts, R. Mols, T.D. Thi, J.A. Martens, J. Van Humbeeck, P. Annaert, G. Van den Mooter, P. Augustijns, Eur. J. Pharm. Sci. 41, 623-630 (2010) 